\title{
MALAT1 Activates the P53 Signaling Pathway by Regulating MDM2 to Promote Ischemic Stroke
}

\author{
Ting Zhang ${ }^{a}$ Hongmei Wang ${ }^{a}$ Qiang Lib Jianliang Fu ${ }^{a} \quad$ Jiankang Huang ${ }^{a}$ \\ Yuwu Zhao ${ }^{a}$ \\ aDepartment of Neurology, Shanghai Jiao Tong University Affiliated Sixth People's Hospital, Shanghai, \\ bDepartment of Neurology, Shanghai Jiao Tong University Affillilated Ninth People's Hospital, \\ Shanghai, China
}

\section{Key Words}

Ischemic stroke • MALAT1 • MDM2 • P53 pathway

\begin{abstract}
Background/Aims: This study focused on evaluating the effect of MALAT1 and MDM2 on ischemic stroke through regulation of the p53 signaling pathway. Materials: Bioinformatics analysis was performed to identify abnormally expressed IncRNAs, mRNAs and their associated pathways. Oxygen-glucose deprivation/reoxygenation (OGD/R) in cells and middle cerebral artery occlusion/reperfusion (MCAO/R) in mice were performed to simulate an ischemic stroke environment. Western blot and qRT-PCR were used to examine IncRNA expression and mRNA levels. Fluorescence in situ hybridization (FISH) LncRNA was used to locate mRNA. MTT and flow cytometry were performed to examine cell proliferation and apoptosis. Finally, immunohistochemistry was used to observe the expression of genes in vivo. Results: MALAT1 and MDM2, which exhibit strong expression in stroke tissues, were subjected to bioinformatics analysis, and the $\mathrm{p} 53$ pathway was chosen for further study. MALAT1, MDM2 and p53 signaling pathway-related proteins were all up regulated in OGD/R cells. Furthermore, Malat1, Mdm2 and p53 pathway related-proteins were also up regulated in MCAO/R mice. Both MALAT1 and MDM2 were localized in the nuclei. Down regulation of MALAT1 and MDM2 enhanced cell proliferation ability and reduced apoptosis, resulting in decreased infarct size in MCAO/R brains. Conclusion: These results indicate that MALAT1/MDM2/p53 signaling pathway axis may provide more effective clinical therapeutic strategy for patients with ischemic stroke.
\end{abstract}

Ting Zhang

and Yuwu Zhao 


\section{Introduction}

Strokes are divided into ischemic and hemorrhagic categories. The former results from a permanent or transient local reduction of cerebral blood supply characterized by cellular disturbances, while the latter is caused by a rupture of blood vessel/s. Approximately $85 \%$ of strokes are ischemic in origin. As a result, the affected area of the brain cannot function properly, resulting in difficulty moving limbs or inability to understand or speak [1]. Ischemic stroke is a complex genetic disorder caused by a combination of multiple genetic and environmental factors. The current strategy for studying stroke pathogenesis is to identify relevant genes early in the process and then to identify the function of those genes [2]. Currently, acute therapeutic intervention for ischemic stroke includes thrombolytic therapy, which is effective only within a narrow time window. Hence, there is an urgent need to develop new and effective therapies [3].

Long non-coding RNAs have many biological roles, such as transcriptional and posttranscriptional regulation, which have been studied in connection with multiple human diseases [4]. In addition, lncRNAs have been shown to play roles in human stroke vascular risk factors like atherosclerosis. One study demonstrated abnormal expression of lncRNAs in stroke, some of which bound to co-repressors of REST, a transcription factor that affects stroke-mediated cell death [5]. LncRNAs are potential novel biomarkers that should be explored further for use in the diagnosis, treatment and prognosis of stroke [6]. Metastasisassociated lung adenocarcinoma transcript 1 (MALAT1) is a highly abundant lncRNA that exerts anti-inflammatory and anti-apoptotic roles in the brain microvasculature to reduce ischemia in the cerebral vasculature [7]. Studies have shown that MALAT1 regulates the function of end othelial cells and vessel growth $[8,9]$. However, the function and mechanisms of MALAT1 in response to stroke have not been comprehensively examined.

The mouse double minute 2 homolog (MDM2) gene encodes a nuclear-localized E3 ubiquitin ligase for p53 in mitotic cells, thereby regulating cellular growth, DNA repair, oxidative stress and apoptosis. MDM2 is an oncoprotein that blocks p53 tumor suppressormediated transcriptional transactivation [10]. A deeper understanding of MDM2 regulation will help us to develop new and more effective strategies for ischemic stroke treatment that target MDM2 and activate p53 signaling in cells, owing to the critical role MDM2 plays in human disease.

Cerebral ischemic injury leads to cell death at ischemic sites. Studies have found that the p53 gene is a key regulator in cell death and is implicated in the regulation of cell loss in stroke [11]. Some studies have shown that MEG3 functions as a cell death promoter in ischemia and interacts with p53 functionally and physically to mediate ischemic injury. Intervention in p53 interactions represents a new target for unique therapeutic development in ischemic insults [12]. Therefore, targeting the p53 signaling pathway minimized the detrimental effects after stroke [13]. We speculated that MDM2 and p53 signaling pathway is functional and has therapeutic significance in ischemic stroke treatment.

We evaluated the mechanism of MALAT1 and MDM2 function in ischemic stroke in this study and found that MALAT1 affects ischemic stroke through regulation of MDM2 and the p53 signaling pathway. Our research may facilitate the understanding of ischemic stroke treatment and provide novel treatment strategies for this serious condition.

\section{Materials and Methods}

\section{Identification of differentially expressed lncRNAs and $m R N A s$}

GEO Series Accession Number GSE58294 (https://www.ncbi.nlm.nih.gov/geo/), National Center for Biotechnology Information (NCBI) Gene Expression Omnibus (GEO) provided our gene expression data, including 69 stroke blood samples and 23 normal blood samples. To identify differentially expressed genes between stroke and normal blood, expression profiles of lncRNAs and mRNAs were analyzed by hierarchical clustering using R package. When the fold change threshold exceeded 2 , the adjusted $P$-value was less than 0.05 for screening.

\section{KARGER}




\section{Cellular Physiology Cell Physiol Biochem 2018;50:2216-2228 \begin{tabular}{ll|l} 
and Biochemistry & $\begin{array}{l}\text { DOI: 10.1159/000495083 } \\
\text { Published online: 13 November } 2018\end{array}$ & $\begin{array}{l}\text { C } 2018 \text { The Author(s). Published by S. Karger AG, Basel } \\
\text { www.karger.com/cpb }\end{array}$ \\
\hline
\end{tabular} \\ Zhang et al.: MALAT1/MDM2 Promote Ischemic Stroke}

\section{Gene set enrichment analysis}

Gene set enrichment analysis (GSEA) was performed based on the Kyoto Encyclopedia of Genes and Genomes (KEGG) database. Expression data of total normalized mRNAs were uploaded to GSEA v3.0 software. Afterward, KEGG pathway enrichment analysis was performed. Given the normalized enrichment score (NES), the seven most enriched pathways that were up regulated in stroke or normal blood were identified and presented as a GSEA enrichment plot.

\section{Network construction for IncRNAs and genes}

The node and edge files were formed using $\mathrm{R}$ software with $P$-value set to less than 0.05 and a coefficient factor threshold of 0.7. We established the interaction networks between IncRNAs and pathwayrelated genes that have differential expression between stroke and normal blood using Cytoscape software.

\section{Cell culture}

Human brain microvascular endothelial cells (HBMECs) obtained from the BeNa Culture Collection (Beijing, China) were plated in 96-well plates at a density of $5 \times 10^{4}$ cells/well and cultured in high-glucose Dulbecco's Modified Eagle Medium (DMEM, $4.5 \mathrm{~g} / \mathrm{L}$ glucose) supplemented with $4 \mathrm{mM}$ glutamine, sodium pyruvate (Sigma, St. Louis, MO, USA) and $10 \%$ fetal bovine serum (Gibco, Rockville, MD, USA) at $37^{\circ} \mathrm{C}$ under $5 \% \mathrm{CO}_{2}$.

\section{Cell transfection}

MALAT1, MDM2, Malat1, Mdm2 and negative control (NC) siRNA were purchased from Santa Cruz Biotechnology (Santa Cruz, CA, USA). MALAT1, MDM2, Malat1, Mdm2 and NC siRNA were synthesized and annealed (Ambion Research, Shanghai, China) for knock down. In line with the manufacturer's recommended protocol, cells were transfected with MALAT1, MDM2, Malat1, Mdm2 and NC siRNA using Lipofectamine 2000 (Invitrogen, Carlsbad, CA, USA). Cells were cultured, and levels of MALAT1, MDM2, Malat1 and Mdm2 were examined $48 \mathrm{~h}$ post-transfection.

\section{Oxygen glucose deprivation/reoxygenation $(O G D / R)$}

Cells were treated with OGD/R exposure as previously described [14]. Briefly, balanced salt solution was adopted to wash cells twice, which were then subjected to OGD medium without glucose (in mM: $\mathrm{NaCl}$ $116, \mathrm{CaCl}_{2} 0.9, \mathrm{MgSO}_{4} 0.8, \mathrm{KCl} 5.4, \mathrm{NaH}_{2} \mathrm{PO}_{4} 1$, phenol red 110, pH 7.4). Later, cells were placed in an anaerobic chamber containing $94 \% \mathrm{~N}_{2}$ and $5 \% \mathrm{CO}_{2}$ at $37^{\circ} \mathrm{C}$ for $2 \mathrm{~h}$. After adding $5 \mathrm{mM}$ glucose to the medium, cells were returned to full culture medium and normoxia for $24 \mathrm{~h}$. Control cells were cultured in a regular $5 \% \mathrm{CO}_{2}$ incubator containing $5 \mathrm{mM}$ glucose at $37^{\circ} \mathrm{C}$ in the same buffer.

\section{$R T-q P C R$}

Total RNA was isolated from cells and tissue. According to the manufacturer's instructions for the reverse transcriptase kit (Takara, Dalian, China), one microgram of extracted RNA was transcribed into cDNA after assessing sample purity using a Thermo Scientific NanoDrop 2000 (MA, USA). Relative quantitative values were determined using the $2^{-\Delta \Delta \mathrm{Ct}}$ method, and Histone 3.1 and GAPDH expression was utilized as internal controls. Primers used for RNA quantification were designed as shown in Table 1.

\section{Western blot}

Total protein was extracted from cells grown in complete medium, and supernatants were collected from cells and tissues [15]. Proteins were separated by $12 \%$ SDS-PAGE and transferred to nitrocellulose filter membranes, which were probed overnight at $4{ }^{\circ} \mathrm{C}$ with the following antibodies: anti-MDM2 (1:1000, ab38618), anti-p53 (phosopho ser15) (1:500, ab1431), and antiBax (1:1000, ab182734) (Abcam, USA).

Table 1. Primers

\begin{tabular}{lc}
\hline Gene & Sequence $\left(5^{\prime}-3^{\prime}\right)$ \\
\hline MALAT1 Forward & GCTCTGTGGTGTGGGATTGA \\
MALAT1 Reverse & GTGGCAAAATGGCGGACTTT \\
Malat1 Forward & GTATGTAGGCCTTTGCGGGT \\
Malat1 Reverse & GGTTGTGCTGGCTCTACCAT \\
MDM2 Forward & GAATCATCGGACTCAGGTACATC \\
MDM2 Reverse & TCTGTCTCACTAATTGCTCTCCT \\
Mdm2 Forward & GAGAGGTTGCAAGATGGCCT \\
Mdm2 Reverse & ACAGAGCCATGGGTGTTCAG \\
\hline
\end{tabular}




\section{Cellular Physiology Cell Physiol Biochem 2018;50:2216-2228

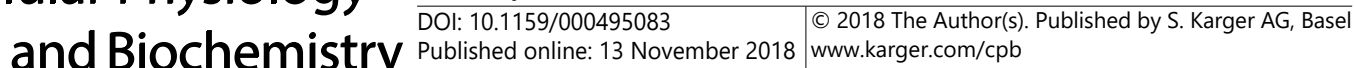 \\ Zhang et al.: MALAT1/MDM2 Promote Ischemic Stroke}

Fluorescence in situ hybridization (FISH) assay

mRNA and LncRNA FISH was performed using Ribo $^{\mathrm{TM}}$ lncRNA FISH Probe Mix (Red) (C10920, RIBOBIO, Shanghai, China). The trial was fulfilled according to the previous description [16]. In short, after preheating 4- $\mu$ m paraffin-embedded tissue sections and formalin fixation, they were incubated with proteases before being hybridized. Next, $20 \mu \mathrm{l}$ Cy3-MALAT1 and Cy3-MDM2 probes were treated with hybridization in a sequential way. In the nuclei, marked as bright red spots, specific staining signals are highlighted.

MTT

After 0 h, 12 h, 24 h or 48 h, $20 \mu \mathrm{l}$ MTT ( 5 mg/ml; Sigma, United States) was added to each well and incubated for a further $4 \mathrm{~h}$. Then, $100 \mu \mathrm{DMSO}$ was added to dissolve the dark blue formazan crystals after careful removal of the media. Finally, optical absorbance was read at $570 \mathrm{~nm}$ by an automatic enzyme-linked immune detector (Bio-Rad Laboratories, Ltd, China).

\section{Flow cytometry}

Apoptosis in response to OGD/R was evaluated with Annexin V fluorescein isothiocyanate (FITC) / apoptosis detection kit (Sigma, San Francisco, CA, USA). Cells were collected by trypsinization after $24 \mathrm{~h}$ OGD/R or non-OGD/R and re-terminated in a binding buffer. Cell suspensions were exposed to $5 \mu \mathrm{l}$ annexin V-FITC and $10 \mu \mathrm{l}$ propidium iodide for $15 \mathrm{~min}$ at $15-25^{\circ} \mathrm{C}$ Stained cells were carefully analyzed using a BD FACS Calibur $^{\mathrm{TM}}$ flow cytometer (BD Biosciences, Piscataway, NJ, USA).

\section{Middle cerebral artery occlusion/reperfusion (MCAO/R)}

$\mathrm{MCAO} / \mathrm{R}$ operation was performed in line with the procedures mentioned above with slight alterations [17]. The absence of corneal reflex and the level and stability of the mean arterial pressure (MAP) jointly determined the proper limits of anesthesia, while supplemental intraperitoneal injection (i. p.) injection of pentobarbital sodium provided as entailed controlled the appropriate levels of anesthesia and analgesia. The left common carotid artery was congested, and the branches of the external carotid artery were dissected and separated. The internal carotid artery was followed rostrally with a 4-0 filament (Beijing Shadong Biology Company, Beijing PR China), containing a diameter of 0.25 and a tip diameter of $0.34 \mathrm{~mm}$ to create a globular stopper, was introduced into the internal carotid artery and advanced until resistance was felt. The filament was removed after $2 \mathrm{~h}$, and mice were placed in a controlled temperature condition $\left(24^{\circ} \mathrm{C}\right.$ to $\left.25^{\circ} \mathrm{C}\right)$ for the first $24 \mathrm{~h}$ after surgery.

TTC

The mouse brains were removed promptly after anesthetized with sodium pentobarbital ( $40 \mathrm{mg} / \mathrm{kg}$ ) by intraperitoneal injection after reperfusion, and their total wet weight was determined. Brains were cut at the forebrain $3 \mathrm{~mm}$, with each brain sliced into six coronal sections of 2-mm thickness, stained with a $2 \%$ solution of tetrazolium chloride (TTC, Sigma, Shanghai, PR China) in saline at $37^{\circ} \mathrm{C}$ for 20 minutes, and imaged. Images were digitized with a Compix system computer.

\section{Immunohistochemistry (IHC)}

IHC assays were utilized to detect p-p53, Mdm2, and Bax protein expression in tissue samples with brain tissue treated, using shams as a negative control. An anti-rabbit Mdm2 antibody (1:30, ab38618, Abcam, USA), anti-rabbit Bax antibody (1:50, ab53154, Abcam, USA) and anti-rabbit p-p53 antibody (20 $\mu \mathrm{g} / \mathrm{ml}, \mathrm{ab} 1431$, Abcam, USA) was used for IHC staining. Briefly, IHC procedures were designed as follows. Deparaffinized and rehydrated formalin-fixed and paraffin-embedded (FFPE) sections were soaked in high $\mathrm{pH}$ target retrieval solution in a pressure cooker (PTlink module, DAKO) at $97^{\circ} \mathrm{C}$ for 35 minutes. Sections were then treated with $2.5 \% \mathrm{H}_{2} \mathrm{O}_{2}$ in methanol for 15 minutes prior to incubation with protein blocking solution (PBS with $2 \%$ cold water fish skin gelatin, $1 \%$ casein, $2 \%$ normal goat serum and $0.1 \%$ Tween-20) for 30 minutes. Afterwards, sections were incubated for 60 minutes at room temperature with primary antibody. After washing twice with TBST, sections were incubated with HRP labeled secondary antibody (1:2000, ab205718, Abcam, USA) for 30 minutes. Slides were visualized using DAB substrate-chromagen (K3468, DAKO) after another two washes with TBST. 


\section{Cellular Physiology Cell Physiol Biochem 2018;50:2216-2228

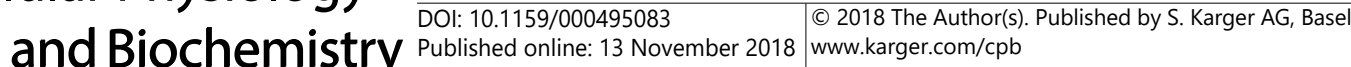 \\ Zhang et al.: MALAT1/MDM2 Promote Ischemic Stroke}

Neurological score

The foot fault test was performed according to published methods [18] to assess sensorimotor function of affected mouse limbs in response to MCAO/R. Animals were trained for three days in a row before surgery, and the score on the day before injury was regarded as baseline. The test was conducted at up to $28 \mathrm{~d}$ after surgery with multitudes of foot fault tests being averaged over three experiments. The total scores of left forelimb and hind limb foot fault tests determined the neurological scores.

\section{Statistical analysis}

Quantitative data are expressed as mean \pm standard deviation (SD). Variance was determined using appropriate post hoc tests (SigmaStat software; Jandel Corporation, San Rafael, CA, USA) via one-way analysis, with comparisons performed among various groups. Student's $t$-test was used for comparisons between two groups, and statistical significance was set at $P<0.05$.

\section{Results}

\section{Microarray analysis}

Differential expression was assessed through microarray analysis for contrast and comparison. Fig. 1A demonstrates a heat map of 20 selected lncRNAs that were significantly differentially expressed compared to the control group. MALAT1 was highly expressed in ischemic stroke samples among the 20 lncRNAs. Similarly, 20 mRNAs with the greatest significant differences from the control group were selected to examine further. The heat map in Fig. 1B illustrates that MDM2 exhibited the highest expression in ischemic stroke among the 20 mRNAs identified.

The p53 signaling pathway is activated in ischemic stroke

Based on the results of GSEA KEGG, the p53 signaling pathway was identified as particularly prominent as assess by the $\mathrm{R}$ package. In contrast to the apelin signaling pathway used as the control group, p53 signaling was significantly activated in stroke, as shown in Fig. 2A. We were able to locate the positions of ranked genes using GSEA KEGG pathway analysis. In the ranked list metric and the running enrichment score shown in Fig. 2B, it was apparent that most genes were up regulated. Results illustrated that the p53 signaling pathway was activated. We then generated a network diagram using Cytoscape to examine differently expressed IncRNAs, mRNAs, related genes of the p53 signaling pathway and their relationship, as shown in Fig. 2C. Results suggested that there is a positive correlation between MALAT1 and MDM2. In Fig. 2D, MDM2 was obtained from intersecting elements from differentially expressed genes in stroke tissues and gene sets of the p53 signaling pathway provided by GSEA, respectively. As the findings indicated, MDM2 not only exhibited differential expression in ischemic stroke tissues, but is also related to the p53 signaling pathway.

MALAT1 and MDM2 exhibit high expression in HBMECs in response to OGD/R treatment

Fig. 3A displays relative expression of MALAT1 and MDM2 in OGD/R HBMECs, with non-GOD/R as internal controls. Expression levels of both MALAT1 and MDM2 in OGD/R HBMECs were up regulated as assessed by RT-qPCR. Western blot was utilized to assess related protein expression of the p53 signaling pathway in OGD/R HBMECs. Results in Fig. 3B illustrate that expression levels of p-p53, MDM2 and BAX were all significantly greater than in controls. Cy3 was employed as a fluorescent label to localize MALAT1 and MDM2 within cells. In Fig. 3C, we merged images of their fluorescent tags, revealing that both of them exhibited nuclear expression. 


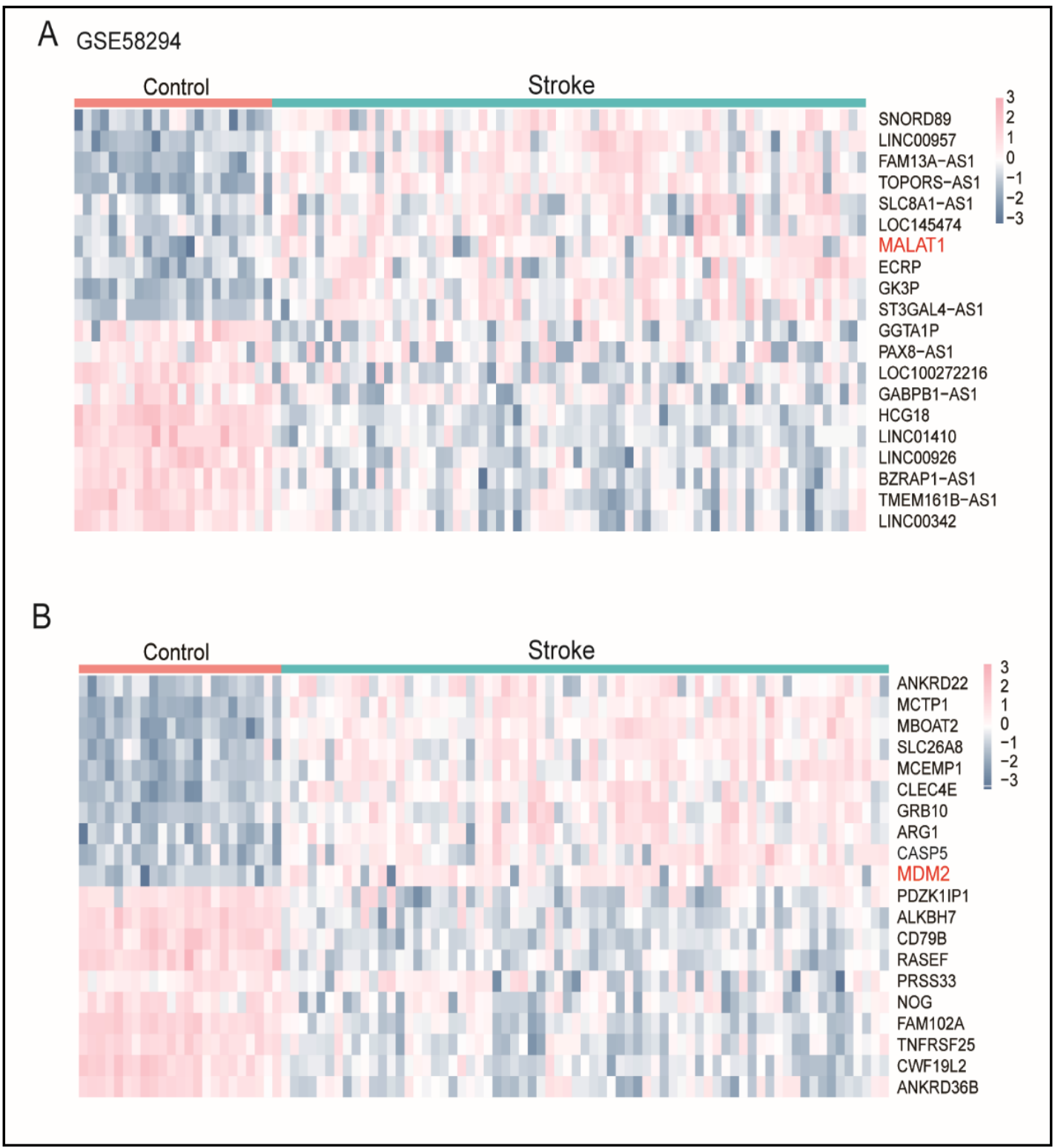

Fig. 1. Microarray analysis. (A). Twenty lncRNAs with the most significant differences were chosen, and a heat map was produced according to their expression. Among them, MALAT1 was most highly expressed in ischemic stroke. (B). Twenty mRNAs that exhibited the most significant differences were chosen, and a heat map was produced according to their expression. Among them, MDM2 was most highly expressed in ischemic stroke.

\section{Growth of HBMECs under different disturbances}

Fig. 4A demonstrates the results of experiments confirming transfection efficiency. Compared to non-OGD/R controls, siMALAT1 effectively reduced the expression of MALAT1 and MDM2, while siMDM2 only decreased expression of MDM2. OGD/R increased expression levels of MALAT1 and MDM2 and weakened siRNA function. In a similar way, other relative expressions of protein were provided by Fig. 4B. After OGD/R, expression of p-p53, MDM2 and BAX were all up regulated. These findings indicate that expression of MDM2, p-p53 and BAX is reduced by siMALAT1 and siMDM2, while OGD/R increased expression levels of these same genes. To examine proliferation of HBMECs in response to these stresses, we performed MTT assays to assess their viability. As shown in Fig. 4C, OGD/R clearly inhibits

\section{KARGER}




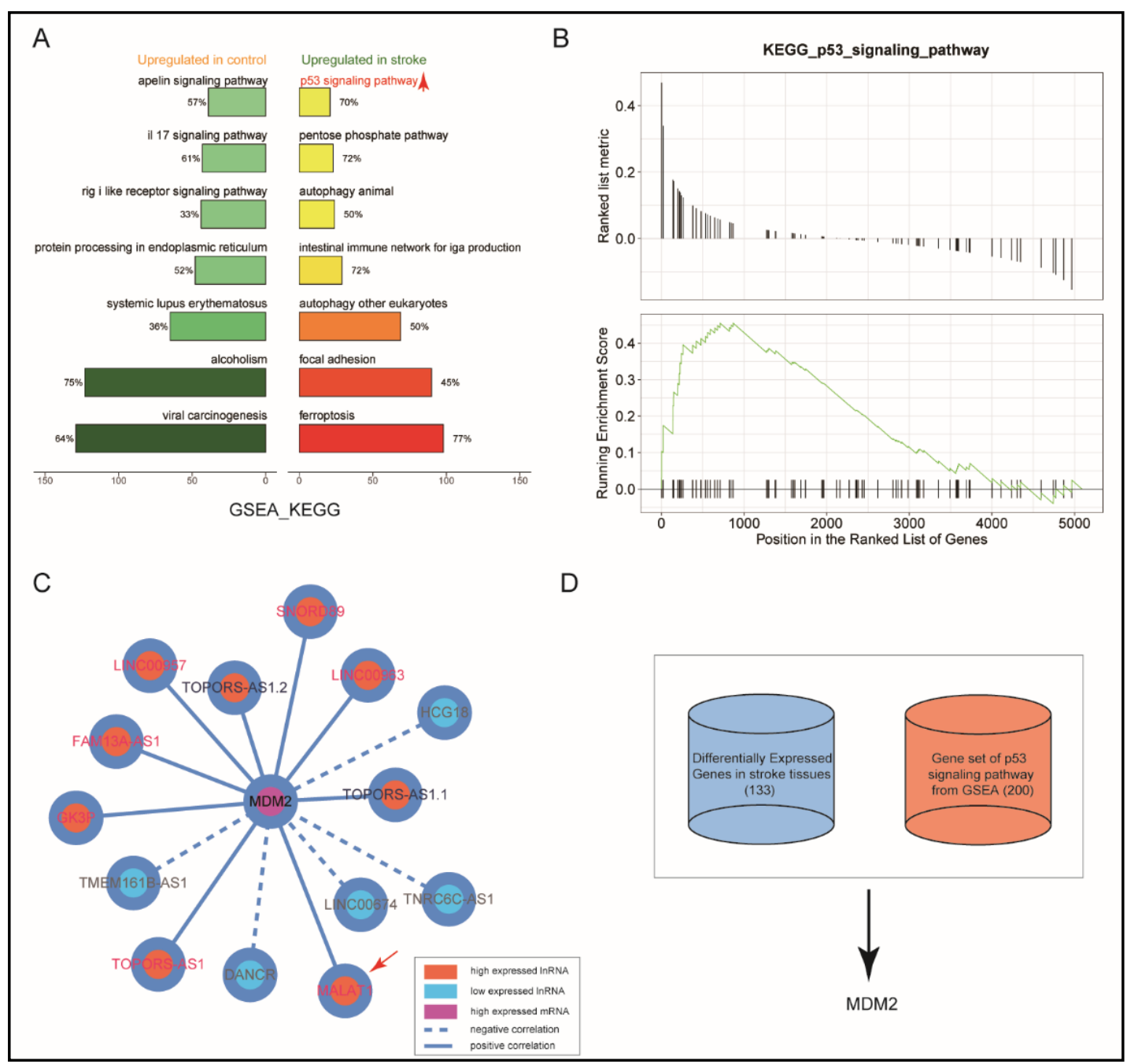

Fig. 2. The p53 signaling pathway is activated in ischemic stroke. (A). Results of GSEA KEGG using the $R$ package. Results showcase that the p53 signaling pathway is activated in ischemic stroke. (B). According to GSEA KEGG analysis results, the p53 signaling pathway is activated. (C). A network diagram illustrating differential expression of IncRNAs and mRNAs produced in Cytoscape. Results illustrate that MALAT1 and MDM2 are positively correlated. (D). MDM2 not only exhibited differential expression in ischemic stroke tissues, but is also related to the p53 signaling pathway.

HBMEC proliferation, while siMALAT1 and siMDM2 promote their proliferation. Apoptosis of HBMECs in response to various disturbances is clearly displayed in Fig. 4D as assessed by flow cytometry. Rates of apoptosis in response to siMALAT1 and siMDM2 were significantly decreased compared to siNC. These results suggest that OGD/R promotes apoptosis, while siMALAT1 and siMDM2 inhibit this effect.

\section{MALAT1 homologous gene IncRNA Malat1 in vivo experiments}

Fig. 5A provides images of mouse brain sections in response to MCAO/R. The white area of the images represents infarct size. Two hours later, we loosened the ligature and collected brain tissue, observing infarct size. Results showed that siMalat1 and siMdm2 decreased size of the damaged area in MCAO/R brains. Fig. 5B illustrates neurological behavior scores. The neurological score of mice exposed to MCAO/R dramatically increased, but siMalat1 and siMdm2 attenuated this process. Expression of Malat1 and Mdm2 in brain tissue was next assessed by RT-qPCR. As shown in Fig. 5C, siMalat1 down regulated their expression levels,

\section{KARGER}




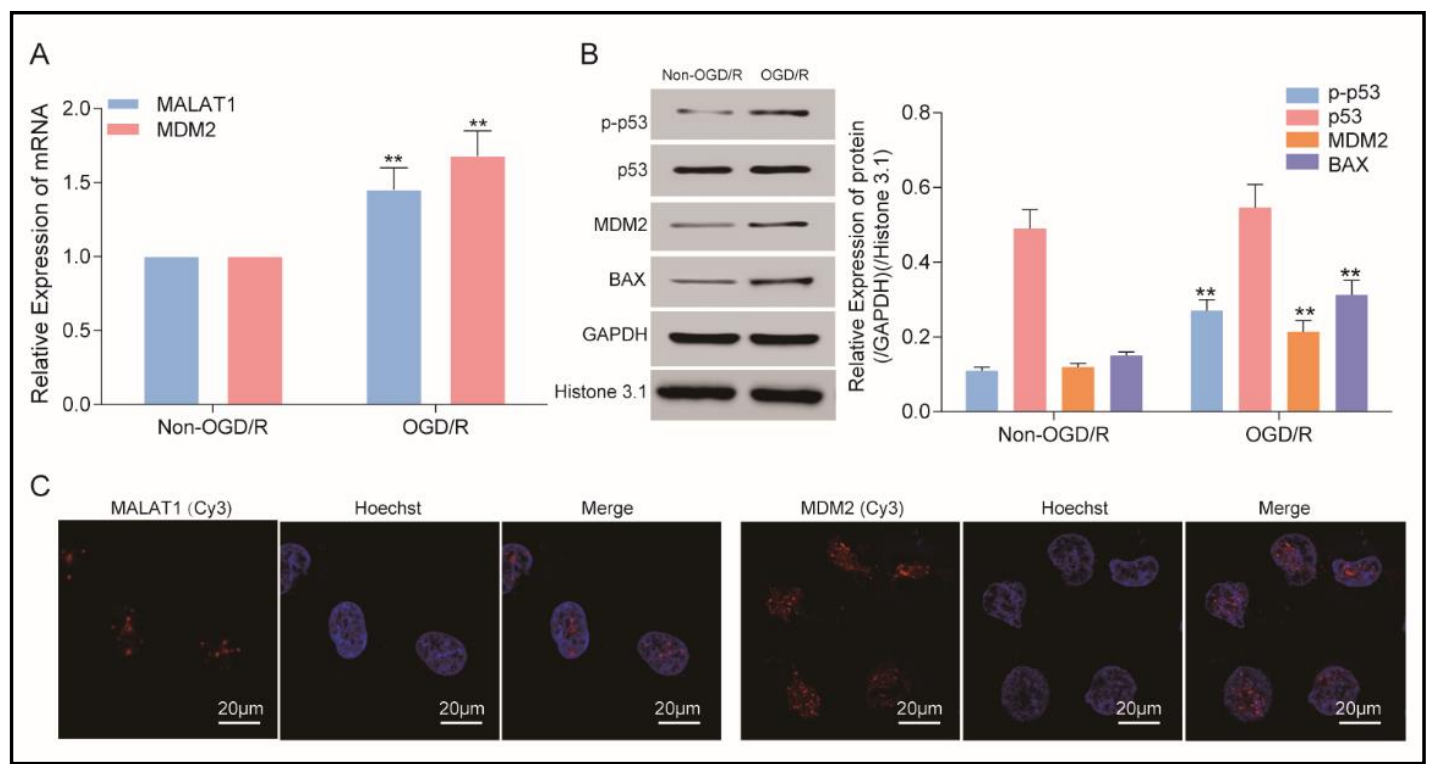

Fig. 3. MALAT1 and MDM2 exhibit high expression in human brain microvascular endothelial cells (HBMECs) after oxygen glucose deprivation/reoxygenation (OGD/R). (A). Expression of MALAT1 and MDM2 in OGD/R HBMECs assessed by RT-qPCR. Results show that their expression levels were both increased. (B). Related protein expression of the p53 signaling pathway in OGD/R HBMECs assessed by western blot. Results illustrate that expression levels of p-p53, MDM2 and BAX increased. (C). MALAT1 and MDM2 subcellular localization results indicate they both exhibit nuclear expression. ${ }^{* *} \mathrm{P}<0.01$, compared to non-OGD/R group.

while siMdm2 down regulated Mdm2's expression level. MCAO/R slowed this process. Western blot was then used to assess expression of $\mathrm{p} 53$ signaling pathway-related proteins in mouse brain tissue. Fig. 5D shows that expression levels of p-p53, Mdm2 and Bax were increased in MCAO/R, while siMalat1 and siMdm2 attenuated these results. Fig. 5E shows brain tissue directly about relative protein expression by immunohistochemistry. Results show that MCAO/R increases expression levels of p-p53, Mdm2 and Bax, while siMalat1 and siMdm2 attenuate this process. 

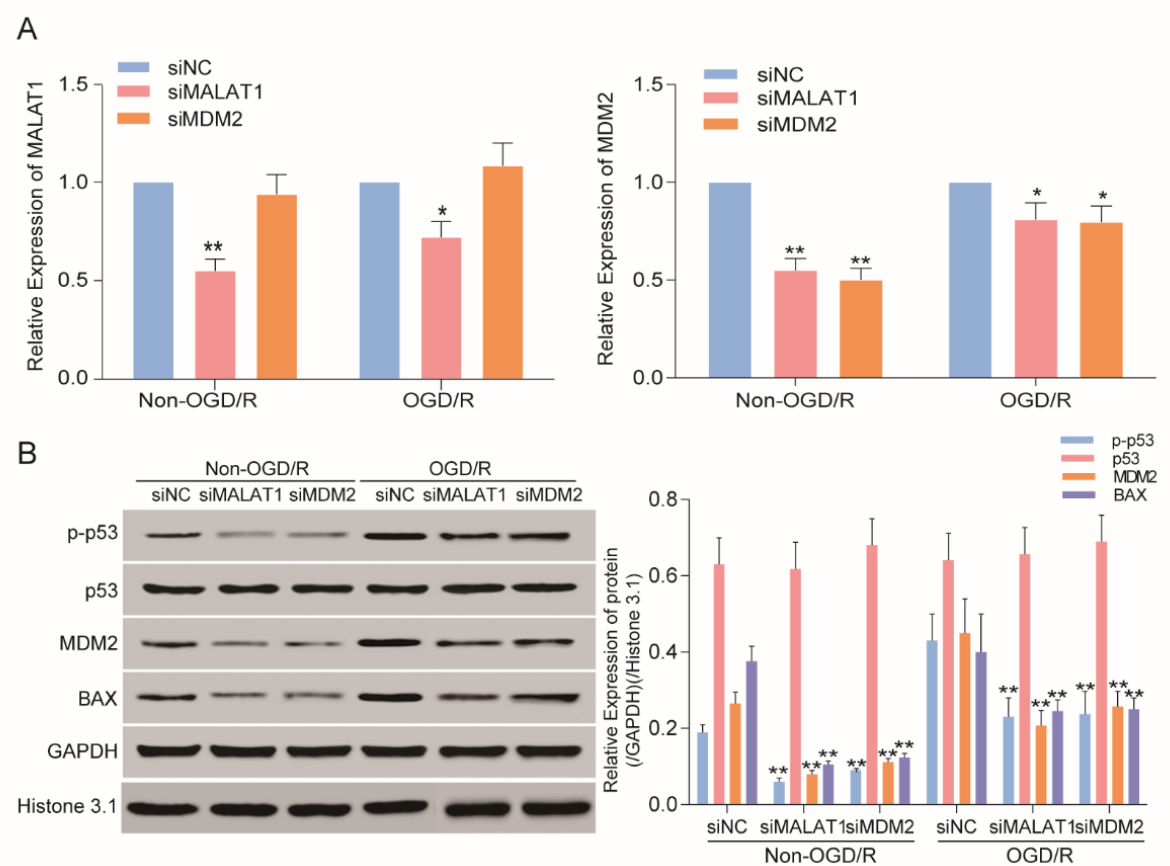

C
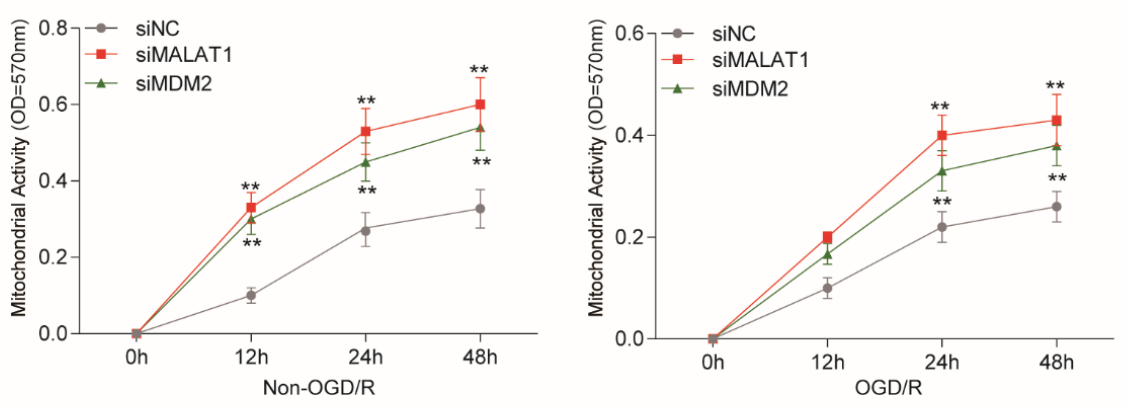

D
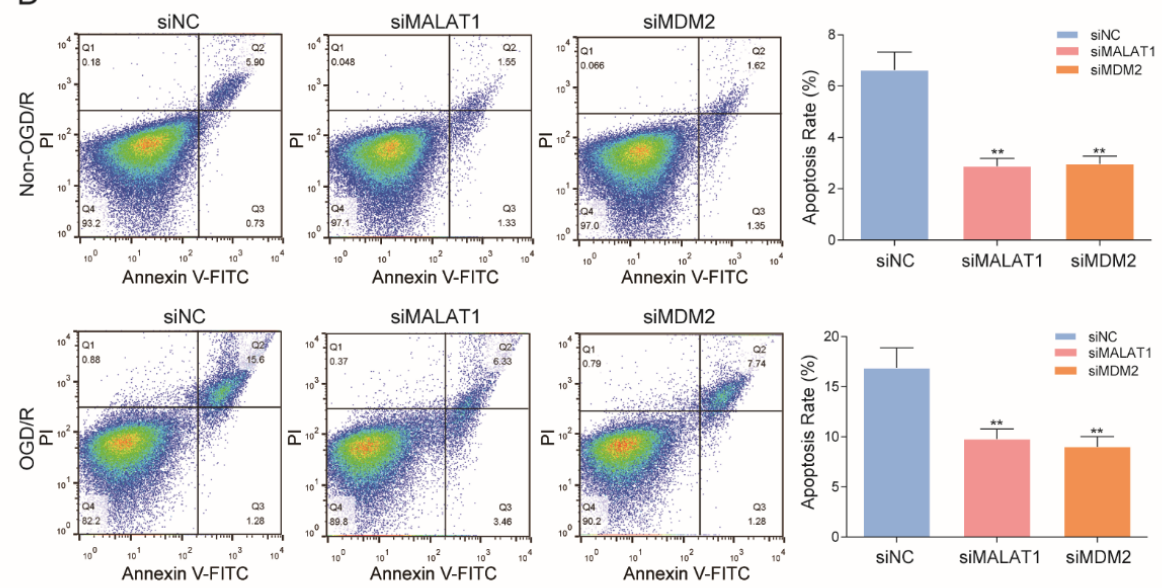

Fig. 4. Growth of HBMECs when different disturbance occurred. (A). Experiment confirming transfection efficiency. (B). Expression levels of MDM2, p-p53 and BAX were down regulated by siMALAT1 and siMDM2, while OGD/R increased their expression levels. (C). Proliferation of HBMECs assessed by MTT assay. Results highlight that OGD/R inhibits its proliferation, while siMALAT1 and siMDM2 promoted its proliferation; (D). Apoptosis of HBMECs in response to various disturbances assessed by flow cytometry. Results suggest that OGD/R promotes apoptosis, while siMALAT1 and siMDM2 inhibit apoptosis. ${ }^{*} \mathrm{P}<0.05$, ${ }^{* *} \mathrm{P}<0.01$, compared to siNC group. 


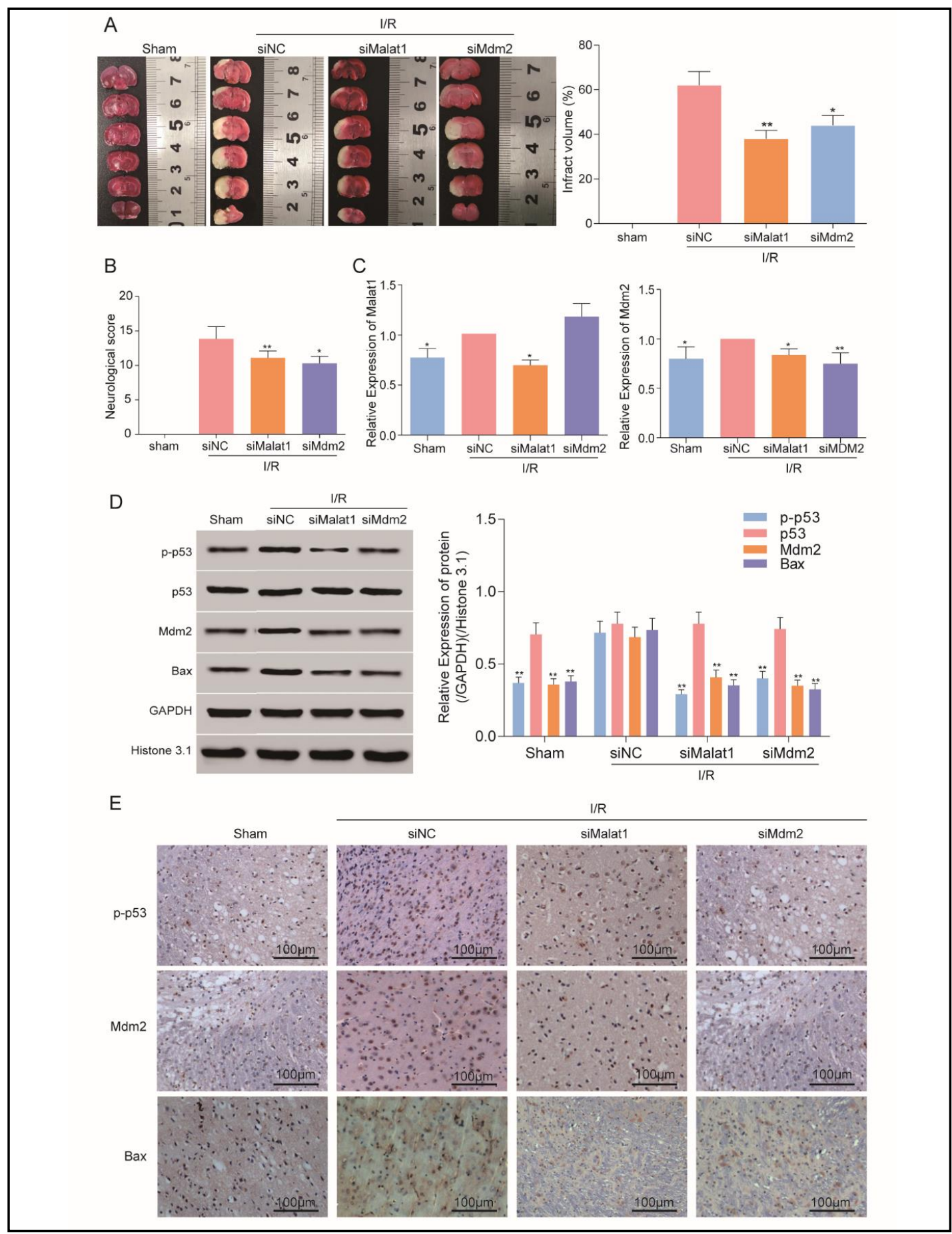

Fig. 5. MALAT1 homologous gene IncRNA Malat1 in vivo experiments. (A). Infarct size in mouse brains after MCAO/R treatment. Results show that siMalat1 and siMdm2 decrease size of the damaged area in the brain caused by MCAO/R. (B). Neurological behavior scores. The neurological score of mice treated with $\mathrm{MCAO} / \mathrm{R}$ rose dramatically, but siMalat1 and siMdm2 attenuated this process. (C). Expression of Malat1 and Mdm2 in brain tissue assessed by RT-qPCR. (D). Expression of p53 signaling pathway-related proteins in mouse brain tissues assessed by western blot. MCAO/R increased expression levels of p-p53, Mdm2 and Bax, while siMalat1 and siMdm2 attenuated this process. (E). Immunohistochemistry results show that MCAO/R increase expression levels of p-p53, Mdm2 and Bax, while siMalat1 and siMdm2 attenuate this process. ${ }^{*} \mathrm{P}<0.05,{ }^{* *} \mathrm{P}<0.01$, compared to siNC group.

\section{KARGER}




\section{Cellular Physiology Cell Physiol Biochem 2018;50:2216-2228 \begin{tabular}{l|l|l} 
and Biochemistry Published online: 13 November 2018 & $\begin{array}{l}\text { (c) } 2018 \text { The Author(s). Published by S. Karger AG, Basel } \\
\text { www.karger.com/cpb }\end{array}$ \\
\hline
\end{tabular} \\ Zhang et al.: MALAT1/MDM2 Promote Ischemic Stroke}

\section{Discussion}

Ischemic stroke is a disease with high mortality, arousing significant attention to improve its treatment [19]. We found that MALAT1 and MDM2 are highly expressed in stroke tissues, which mediates promotion of ischemic stroke via activating the p53 signaling pathway. In addition, Malat1, Mdm2 and $\mathrm{p} 53$ pathway related-proteins were also up regulated in MCAO/R mice. Down regulation of MALAT1 and MDM2 enhanced cell proliferation capacity and further reduced apoptosis. Nevertheless, down regulation of Malat1 and Mdm2 decreased infarct size of MCAO/R brains. Our results illustrated that MALAT1 and MDM2 are novel markers and potential therapeutic targets for ischemic stroke.

MALAT1 has been identified in multiple physiological processes, including epigenetic modulation of gene expression. Abundant evidence indicates that MALAT1 is closely related to various pathological processes, ranging from diabetes complications to cancers [20, 21]. Up regulated MALAT1 expression is correlated with poor overall survival in various cancers $[4,22]$. We found that its expression was up regulated in stroke tissues in our study in accordance with results from Guo et al. [23]. Furthermore, we demonstrated that down regulation of MALAT1 suppresses ischemic injury both in vitro and in vivo, further attenuating neuronal cell death in cerebral ischemic stroke. Recently, more advances are focusing on the neuroprotective effects of ncRNAs in ischemic stroke $[5,12]$, helping to better understand the pathogenesis and progression of ischemic stroke. Although the biological role of MALAT1 in ischemic stroke has been explained, the precise regulatory mechanism by which MALAT1 modulates ischemic stroke remains largely unknown. Our study has demonstrated that expression of MDM2 is directly regulated by MALAT1. Moreover, Malat1, Mdm2 and p53 pathway-related proteins were also up regulated in MCAO/R mice, consistent with previous findings $[24,25]$. Overall, these results reveal that up regulation of MALAT1 exerts its biological roles through regulation of MDM2 expression in ischemic stroke. A previous study indicated that Malat1 plays anti-apoptotic and anti-inflammatory roles in the brain microvasculature to reduce ischemic stroke [7]. In addition, studies have uncovered the regulation pattern of Malat1 expression mediated by p53 in hematopoietic differentiation [26]. In keeping with our study, these results indicate that MALAT1 mediates ischemic stroke via activating the $\mathrm{p} 53$ signaling pathway.

Many studies have shown that MDM2 can enhance p53 activity [27, 28]. Moreover, previous investigation has revealed that p53 is a key modulator of cellular stress responses, becoming activated in ischemic areas of the brain and contributing to neuronal apoptosis. p53 deficiency or applications using p53 inhibitors attenuate brain damage significantly in various stroke models $[29,30]$. Surprisingly, we found that MDM2 was strongly expressed in stroke tissues, which promoted ischemic stroke via activating the p53 signaling pathway. Hereby, a mechanism connecting ischemic stroke and MDM2 has been confirmed. Previous studies have confirmed our findings that p53 inhibition has a key protective effect against ischemia-reperfusion injury in vitro and provides a promising therapeutic target against stroke [31,32].

Despite these findings, our study still has some limitations. For example, detailed investigation of genes that comprise the MALAT1/MDM2/p53 signaling pathway axis should yield further insights into the mechanism by which MALAT1 induces ischemic stroke progression. Moreover, the exact role of MALAT1/MDM2/p53 signaling pathways in ischemic stroke still needs further exploration. Nevertheless, our results indicate that MALAT1 and MDM2 are novel markers and potential therapeutic targets for ischemic stroke. 


\section{Cellular Physiology Cell Physiol Biochem 2018;50:2216-2228 \begin{tabular}{ll|l} 
and BiOchemistry & $\begin{array}{l}\text { DOI: 10.1159/000495083 } \\
\text { Published online: 13 November } 2018\end{array}$ & $\begin{array}{l}\text { (c) } 2018 \text { The Author(s). Published by S. Karger AG, Basel } \\
\text { www.karger.com/cpb }\end{array}$ \\
\hline
\end{tabular} \\ Zhang et al.: MALAT1/MDM2 Promote Ischemic Stroke}

\section{Conclusion}

We identified that expression of MALAT1 and MDM2 are increased in stroke tissues and cells. Upon exploring the mechanism of stroke progression regulated by MALAT1, we demonstrated that MALAT1 regulates MDM2 and facilitates its expression, further repressing stroke cell proliferation and increasing apoptosis. In contrast, MALAT1 activates p53 signaling and up regulates Malat1, Mdm2 and p53 pathway-related proteins. In conclusion, MALAT1 significantly contributes to ischemic stroke development by regulating MDM2 and subsequently activating the p53 signaling pathway. These results indicate that the MALAT1/ MDM2/p53 signaling pathway axis may represent a more effective clinical therapeutic strategy for patients with ischemic stroke.

\section{Acknowledgements}

This work was supported by Medical Professional Cross Research Fund Project of Shanghai Jiao Tong University (YG2015MS14), 2016 Shanghai Jiaotong University Type-C Subsidy of Chenxing Award Project, and the Grants from National Natural Science Foundation of China (81672243) \& (31271125).

\section{Disclosure Statement}

The authors have no conflicts of interest to declare.

\section{References}

$>1$ Chaturvedi M, Kaczmarek L: Mmp-9 inhibition: a therapeutic strategy in ischemic stroke. Mol Neurobiol 2014;49:563-573.

2 Cheng YC, Cole JW, Kittner SJ, Mitchell BD: Genetics of ischemic stroke in young adults. Circ Cardiovasc Genet 2014;7:383-392.

-3 Yin KJ, Hamblin M, Chen YE: Angiogenesis-regulating microRNAs and Ischemic Stroke. Curr Vasc Pharmacol 2015;13:352-365.

4 Tian X, Xu G: Clinical value of IncRNA MALAT1 as a prognostic marker in human cancer: systematic review and meta-analysis. BMJ Open 2015;5:e008653.

$>5$ Saugstad JA: Non-Coding RNAs in Stroke and Neuroprotection. Front Neurol 2015;6:50.

-6 Dykstra-Aiello C, Jickling GC, Ander BP, Shroff N, Zhan X, Liu D, Hull H, Orantia M, Stamova BS, Sharp FR: Altered Expression of Long Noncoding RNAs in Blood After Ischemic Stroke and Proximity to Putative Stroke Risk Loci. Stroke 2016;47:2896-2903.

-7 Zhang X, Tang X, Liu K, Hamblin MH, Yin KJ: Long Noncoding RNA Malat1 Regulates Cerebrovascular Pathologies in Ischemic Stroke. J Neurosci 2017;37:1797-1806.

-8 Yang H, Xi X, Zhao B, Su Z, Wang Z: KLF4 protects brain microvascular endothelial cells from ischemic stroke induced apoptosis by transcriptionally activating MALAT1. Biochem Biophys Res Commun 2018;495:2376-2382.

9 Michalik KM, You X, Manavski Y, Doddaballapur A, Zornig M, Braun T, John D, Ponomareva Y, Chen W, Uchida S, Boon RA, Dimmeler S: Long noncoding RNA MALAT1 regulates endothelial cell function and vessel growth. Circ Res 2014;114:1389-1397.

10 Mendoza M, Mandani G, Momand J: The MDM2 gene family. Biomol Concepts 2014;5:9-19.

11 Filichia E, Shen H, Zhou X, Qi X, Jin K, Greig N, Hoffer B, Luo Y: Forebrain neuronal specific ablation of p53 gene provides protection in a cortical ischemic stroke model. Neuroscience 2015;295:1-10.

-12 Yan H, Yuan J, Gao L, Rao J, Hu J: Long noncoding RNA MEG3 activation of p53 mediates ischemic neuronal death in stroke. Neuroscience 2016;337:191-199. 


\section{Cellular Physiology Cell Physiol Biochem 2018;50:2216-2228 \begin{tabular}{l|l|l|l} 
and BOl: 10.1159/000495083 & $\begin{array}{l}\text { @ } 2018 \text { The Author(s). Published by S. Karger AG, Basel } \\
\text { www.karger.com/cpb }\end{array}$
\end{tabular} \\ Zhang et al.: MALAT1/MDM2 Promote Ischemic Stroke}

13 Venna VR, Verma R, O’Keefe LM, Xu Y, Crapser J, Friedler B, McCullough LD: Inhibition of mitochondrial p53 abolishes the detrimental effects of social isolation on ischemic brain injury. Stroke 2014;45:3101-3104.

14 Mo ZT, Fang YQ He YP, Zhang S: beta-Asarone protects PC12 cells against OGD/R-induced injury via attenuating Beclin-1-dependent autophagy. Acta Pharmacol Sin 2012;33:737-742.

15 Wang HC, Yang Y, Xu SY, Peng J, Jiang JH, Li CY: The CRISPR/Cas system inhibited the pro-oncogenic effects of alternatively spliced fibronectin extra domain A via editing the genome in salivary adenoid cystic carcinoma cells. Oral Dis 2015;21:608-618.

16 Bishop JA, Ma XJ, Wang H, Luo Y, Illei PB, Begum S, Taube JM, Koch WM, Westra WH: Detection of transcriptionally active high-risk HPV in patients with head and neck squamous cell carcinoma as visualized by a novel E6/E7 mRNA in situ hybridization method. Am J Surg Pathol 2012;36:1874-1882.

17 Jiang WL, Zhang SP, Zhu HB, Jian H, Tian JW: Cornin ameliorates cerebral infarction in rats by antioxidant action and stabilization of mitochondrial function. Phytother Res 2010;24:547-552.

18 Metz GA, Whishaw IQ: Cortical and subcortical lesions impair skilled walking in the ladder rung walking test: a new task to evaluate fore- and hindlimb stepping, placing, and co-ordination. J Neurosci Methods 2002;115:169-179.

19 Mortality GBD, Causes of Death C: Global, regional, and national life expectancy, all-cause mortality, and cause-specific mortality for 249 causes of death, 1980-2015: a systematic analysis for the Global Burden of Disease Study 2015. Lancet 2016;388:1459-1544.

20 Yoshimoto R, Mayeda A, Yoshida M, Nakagawa S: MALAT1 long non-coding RNA in cancer. Biochim Biophys Acta 2016;1859:192-199.

21 Wu Y, Huang C, Meng X, Li J: Long Noncoding RNA MALAT1: Insights into its Biogenesis and Implications in Human Disease. Curr Pharm Des 2015;21:5017-5028.

22 Wei Y, Niu B: Role of MALAT1 as a Prognostic Factor for Survival in Various Cancers: A Systematic Review of the Literature with Meta-Analysis. Dis Markers 2015;2015:164635.

-23 Guo D, Ma J, Yan L, Li T, Li Z, Han X, Shui S: Down-Regulation of Lncrna MALAT1 Attenuates Neuronal Cell Death Through Suppressing Beclin1-Dependent Autophagy by Regulating Mir-30a in Cerebral Ischemic Stroke. Cell Physiol Biochem 2017;43:182-194.

24 Yu F, Lu Z, Cai J, Huang K, Chen B, Li G, Dong P, Zheng J: MALAT1 functions as a competing endogenous RNA to mediate Rac1 expression by sequestering miR-101b in liver fibrosis. Cell Cycle 2015;14:3885-3896.

-25 Wang F, Ying HQ, He BS, Pan YQ, Deng QW, Sun HL, Chen J, Liu X, Wang SK: Upregulated IncRNA-UCA1 contributes to progression of hepatocellular carcinoma through inhibition of miR-216b and activation of FGFR1/ERK signaling pathway. Oncotarget 2015;6:7899-7917.

26 Ma XY, Wang JH, Wang JL, Ma CX, Wang XC, Liu FS: Malat1 as an evolutionarily conserved lncRNA, plays a positive role in regulating proliferation and maintaining undifferentiated status of early-stage hematopoietic cells. BMC Genomics 2015;16:676.

27 Tian H, Tackmann NR, Jin A, Zheng J, Zhang Y: Inactivation of the MDM2 RING domain enhances p53 transcriptional activity in mice. J Biol Chem 2017;292:21614-21622.

-28 Saito A, Hayashi T, Okuno S, Nishi T, Chan PH: Modulation of p53 degradation via MDM2-mediated ubiquitylation and the ubiquitin-proteasome system during reperfusion after stroke: role of oxidative stress. J Cereb Blood Flow Metab 2005;25:267-280.

29 Leak RK, Zhang L, Luo Y, Li P, Zhao H, Liu X, Ling F, Jia J, Chen J, Ji X: Peroxiredoxin 2 battles poly(ADPribose) polymerase 1- and p53-dependent prodeath pathways after ischemic injury. Stroke 2013;44:11241134.

-30 Hong LZ, Zhao XY, Zhang HL: p53-mediated neuronal cell death in ischemic brain injury. Neurosci Bull 2010;26:232-240.

31 Xu ZQ Yang MG, Liu HJ, Su CQ: Circular RNA hsa_circ_0003221 (circPTK2) promotes the proliferation and migration of bladder cancer cells. J Cell Biochem 2018;119:3317-3325.

-32 Li X, Gu S, Ling Y, Shen C, Cao X, Xie R: p53 inhibition provides a pivotal protective effect against ischemiareperfusion injury in vitro via mTOR signaling. Brain Res 2015;1605:31-38. 